\title{
Beyond the usual suspects
}

DOI:

10.1038/nrd2301
Profiling cancer mutations aims at providing a molecular portrait of each individual tumour sample to aid clinicians in improving diagnosis and tailoring the best therapy to each patient. However, the road to the clinic is not free from hurdles because of the cost and complexity of identifying the mutations that contribute to each cancer. Two recent studies have made important steps towards resolving these problems.

Given that mutations in oncogenes do not occur randomly but are more frequent in certain regions, Levi Garraway and colleagues decided to focus on these regions, instead of scanning the entire genome of cancer cells. They applied a high-throughput genotyping technique to analyse the frequency and distribution of 238 known mutations - which affect 17 oncogenes - in 1,000 samples derived from 17 different tumour types. One important result is that this genotyping approach, which relies on mass spectrometry,

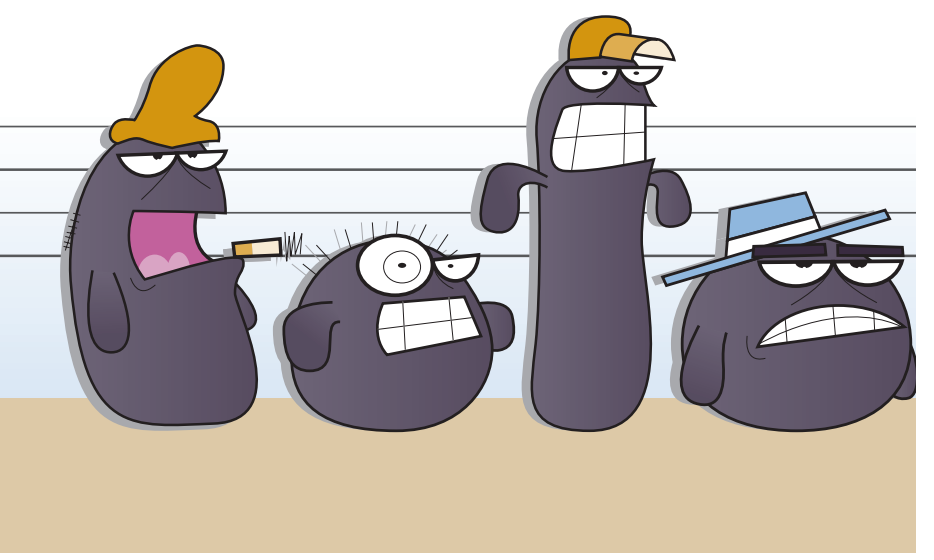

provided mutation profiles that were sensitive and cost-effective compared with the commonly used sequencing approach. Mutations were found in most of the oncogenes that were analysed and their frequency was consistent with previous reports. In addition, mutations were also found in tumour types in which they had not been reported previously, indicating that rare and potentially targetable mutations can be identified with this approach.

Another challenge is to delineate the complexity in cancer genomes. Cancer genomes carry two classes of mutations: 'driver' mutations, which are positively selected because they are essential for tumour growth and development, and 'passenger' mutations, which are not subject to selection because they don't confer a growth advantage. So how can passenger mutations be identified and eventually ruled out from large-scale resequencing screens? Andrew Futreal, Michael Stratton and colleagues set out to survey the number and pattern of somatic mutations in 210 tumour samples of different origin and to establish their relevance to the tumorigenic process. They sequenced the coding exons of 518 protein kinase genes, which are among the most commonly mutated genes in cancer, and identified over 1,000 mutations. Mutational signatures varied widely between tumours depending on cellular origin, DNA-repair ability and previous exposure to carcinogens.

To discriminate between driver and passenger mutations, the authors annotated the 921 single-base substitutions that they identified as being either non-synonymous (changing an amino acid) or synonymous (having no effect on the protein sequence). They proposed that a higher ratio of nonsynonymous to synonymous mutations, compared with that expected by chance, would be the result of positive selection, and would therefore point to the presence of driver mutations. On this basis, 763 mutations were classified as passenger mutations, suggesting that most of the mutations identified through cancer profiling are not implicated in cancer development, even when the screening focuses on a family of genes that are often mutated in cancer. At the same time, the number of driver mutations was larger than previously anticipated and, interestingly, most of the genes with driver mutations were not previously thought to be involved in tumorigenesis, indicating that more cancer genes exist than just the usual suspects.

Altogether, the approaches that these studies have described should open up opportunities for screening the most relevant mutations in tumours efficiently and cost-effectively.

Francesca Pentimalli Nature Reviews Cancer

ORIGINAL RESEARCH PAPERS Thomas, R. K. et al. High-throughput oncogene mutation profiling in human cancer. Nature Genet. 11 February 2007 (doi:10.10138/ng1975) | Greenman, C. et al. Patterns of somatic mutation in human cancer genomes. Nature 8 March 2007 (doi:10.10138/nature05610) 